Int. J. Dev. Biol. 54: 1383-1388 (2010)

doi: $10.1387 / \mathrm{ijdb} .092998 \mathrm{ch}$

\title{
Expression from a $\beta$ geo gene trap in the Slain 1 gene locus is predominantly associated with the developing nervous system
}

\author{
CLAIRE E. HIRST ${ }^{1}$, SUE-MEI LIM ${ }^{1}$, LLOYD A. PEREIRA², ROBYN A. MAYBERRY1, \\ EDOUARD G. STANLEY ${ }^{1}$ and ANDREW G. ELEFANTY*,1

\begin{abstract}
${ }^{1}$ Monash Immunology and Stem Cell Laboratories, Monash University, Clayton, Victoria, Australia and ${ }^{2}$ Differentiation and Transcription Laboratory, Peter MacCallum Cancer Centre, East Melbourne, Victoria,
\end{abstract} \\ Australia.
}

\begin{abstract}
Slain 1 was originally identified as a novel stem cell-associated gene in transcriptional profiling experiments comparing mouse and human embryonic stem cells (ESCs) and their immediate differentiated progeny. In order to obtain further insight into the potential function of Slain 1, we examined the expression of $\beta$-galactosidase in a gene-trap mouse line in which a $\beta g e o$ reporter gene was inserted into the second intron of Slain1. In early stage embryos (E7.5), the Slain 1-ßgeo fusion protein was expressed within the entire epiblast, but by E9.5 became restricted to the developing nervous system and gastrointestinal tract. In later stage embryos (E11.5 - E13.5), expression was predominantly within the developing nervous system. Lower level expression was also observed in the developing limb buds, in the condensing mesenchyme, along the apical epidermal ridge and, at later stages, within the digital zones. These observations suggest that Slain 1 may play a role in the development of the nervous system, as well as in the morphogenesis of several embryonic structures.
\end{abstract}

KEY WORDS: Slain1, mouse, nervous system, sensory ganglia, limb bud development

\section{Introduction}

The Slain family of genes encode proteins that are highly conserved throughout vertebrate evolution. However, other than serine- and proline-rich domains, thought to be involved in protein-protein interactions, neither Slain1 nor Slain2 contain wellrecognized protein motifs that provide clues to their function. Similarly, little is known about the function or expression of the Slaingene family during development. The S/ain 1 gene is located on chromosome 14 and gives rise to 2 alternate transcripts that differ by the inclusion or exclusion of exon 3 (Hirst et al., 2006). In order to gain further insight into the potential function of this novel group of genes, we have undertaken a detailed analysis of the developmental expression of $\beta$-galactosidase from a S/ain 1- $\beta$ geo gene trap mouse line-using X-gal staining of whole mount embryos.

\section{Results and Discussion}

\section{Characterisation of the Slain1- $\beta$ geo insertion}

Two mESC lines, XG385 and XG752, which have the pGT1Lxf gene trap vector integrated into intron B of the mouse Slain 1gene were obtained from the Mutant Mouse Regional Resource Centre (http://www.mmrrc.org/). These lines were predicted to express a protein in which the $\mathrm{N}$-terminus of Slain 1 (encoded by exons 1 and 2 ) was fused with $\beta$ geo ( $\beta$-galactosidase fused with neomycin phosphotransferase; Fig. 1A). Using primers corresponding to sequences located in Slain 1 exon 2 and $\beta g e o$, RT-PCR analysis identified transcription of Slain 1-Bgeo in the bone marrow, brain, kidney, lung, testis and thymus; with lower levels observed in the

Abbreviations used in this paper: ESC, embryonic stem cell.

\footnotetext{
*Address correspondence to: Andrew G Elefanty. Monash Immunology and Stem Cell Laboratories, Building 75, STRIP 1, West Ring Road, Monash University, Clayton, Victoria 3800, Australia. Fax: +61-3-9905-0680. e-mail: andrew.elefanty@med.monash.edu.au
}

Supplementary Material (one figure) for this paper is available at: http://dx.doi.org/10.1387/ijdb.092998ch

Accepted: 6 November 2009. Final author corrected PDF published online: 4 May 2010. Edited by: Christine Mummery.

ISSN: Online 1696-3547, Print 0214-6282

() 2010 UBC Press

Printed in Spain 
A
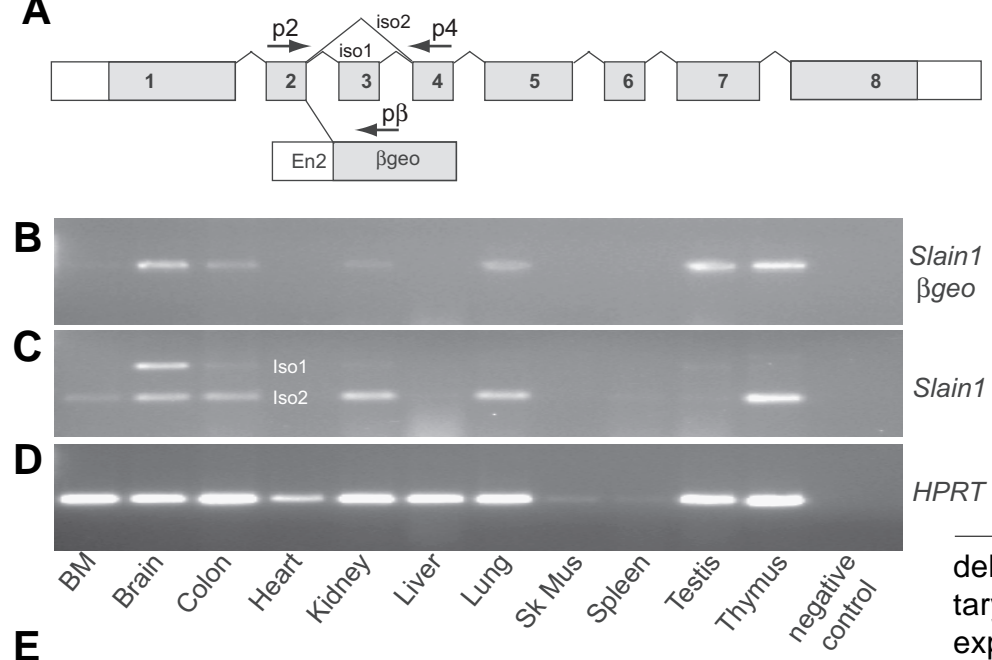

\begin{tabular}{|c|c|c|c|c|c|c|c|c|c|c|l|}
\hline BM & Brain & Colon & Heart & Kidney & Liver & Lung & Sk Mus & Spleen & Testis & Thymus & Splice Form \\
\hline+ & ${ }^{+++}$ & ++ & - & ++ & - & ++ & - & - & ${ }^{++++}$ & ${ }^{+++}$ & Slain1- $\beta g e o$ \\
\hline- & +++ & + & - & + & - & - & - & - & + & + & Slain 1.1 \\
\hline+ & +++ & ${ }^{++}$ & - & +++ & + & ${ }^{+++}$ & - & + & + & +++ & Slain 1.2 \\
\hline
\end{tabular}

colon (Fig. 1B). These data confirmed that Slain1-ßgeo transcripts exhibited the same tissue distribution to that previously described for Slain1 in wildtype mice (Hirst et al., 2006).

Insertion of the $\beta g e o$ cassette into intron $\mathrm{B}$ was predicted to result in the generation of a chimeric transcript in which Slain1 sequences terminated at the end of exon 2. However, analysis of homozygous Slain $\gamma^{\beta g e o} \beta g e o$ mice indicated that transcripts including more distal Slain1sequences were still present; indicating that insertion of the $\beta g e o$ gene trap vector did not create a Slain 1 null allele (Fig. 1C and data not shown). We have previously reported alternative splicing of Slain 1 around exon 3, which generates two isoforms (Hirst et al., 2006). Isoform 1 (which includes exon 3) was most highly expressed in brain, whilst isoform 2 (exon 3
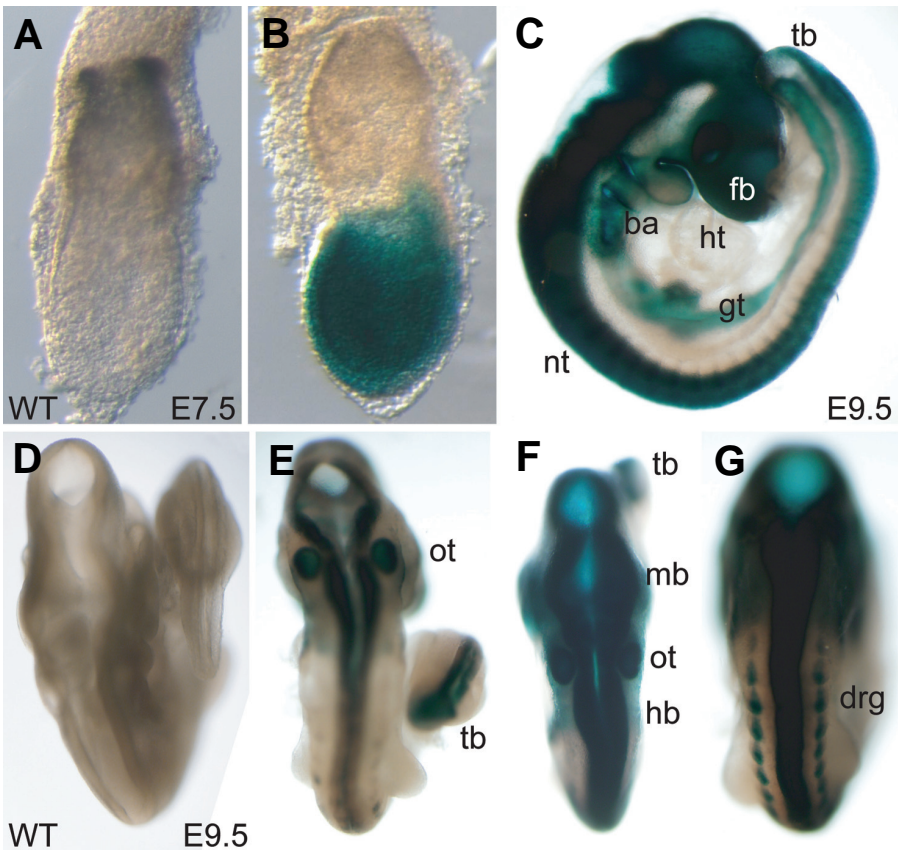

Fig. 1. Analysis of Slain 1- $\beta$ geo transcripts in adult tissues from

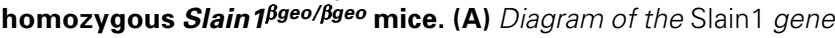
structure indicating the insertion of the En2- $\beta$ geo cassette (containing sequence from intron 1 of the En2 gene and its corresponding splice acceptor site, followed by the fusion of $\beta$ galactosidase with neomycin phosphotransferase) into intron $B$ and positioning of PCR primers. (B-D) Semi-quantitative RT-PCR analysis of Slain1- $\beta$ geo transcripts compared to HPRT expression. (B) Detection of the Slain 1- $\beta$ geo transcripts using primers in exon 2 and $\beta$ geo (primers $p 2$ and $p \beta$ ). (C) Detection of wildtype Slain 1 transcripts using primers in exon 2 and 4 (primers $p 2$ and p4). The PCR products corresponding Slain1 to the alternate transcripts that include (Iso1) and exclude (Iso2) exon 3 are indicated. (E) Summary of the tissue distribution of the splicing forms. The scoring system is based on a subjective assessment of HPRT band intensity from absent (-) to very strong $(++++)$.

deleted) was more widely expressed (Fig. 1C, E and Supplementary Fig. 1). Overall, our analysis suggests that Slain $1 \beta g e ß g e o$ mice express three Slain 1 related transcripts, representing the Slain 1$\beta g e o$ fusion gene and isoforms 1 and 2 . We hypothesise that the isoform 1 resulted from splicing out of the $\beta g e o$ gene trap sequences while isoform 2 arose after splicing out both $\beta g e o$ and exon 3 sequences.

\section{Slain1-ßgeo expression during early embryogenesis}

Expression of Slain1- $\beta$ geo in the mouse embryo was investigated by staining of embryos from timed matings of heterozygous Slain $\beta^{\beta g e o} / \mathrm{w}$ mice for $\beta$ galactosidase activity. There was no observed attrition of homozygous Slain $\beta^{\beta g e o} \beta$ geo embryos during development and heterozygote and homozygote embryos were distinguishable only by a greater intensity of $\beta$ geo staining in the homozygotes. At E7.5, Slain1- $\beta$ geo expression was observed throughout the entire epiblast (Fig. 2B), as predicted by our previous in situ hybridisation (ISH) and embryonic stem cell differentiation data, which indicated that Slain 1 was highly expressed at this stage of mouse development (Hirst et al., 2006).

Consistent with prior ISH studies, whole mount X-gal staining of E9.5 embryos showed robust Slain1- $\beta$ geo expression along the entire neural tube, continuous with the tailbud (Fig. $2 \mathrm{C}$ and F). Expression was also observed in the neural crest-derived dorsal root ganglia (Fig. 2G), as well as in the surface ectoderm of the branchial arches (Fig. 2C) and the otic vesicle (Fig. 2E). In contrast to earlier ISH studies, Slain1- $\beta$ geo was also observed in the primitive gut tube, probably reflecting the greater sensitivity of the X-gal analysis (Fig. 2C).

In E11.5 embryos stained for 4 hours, the strongest areas of Slain $1-\beta g e o$ expression were observed within the forebrain, eye and neural tube (Fig. 3B). Weaker staining was detected within the optic and oropharyngeal regions and parts of the gut tube (Fig. 3B). Overnight staining of embryos revealed additional Slain1-

Fig. 2. Slain1-ßgeo expression in pre- and post-implantation embryos. Photomicrographs of whole mount X-gal stained Slain1- $\beta g e o$ heterozygous (E) and homozygous mouse embryos (B,C,F, G) or wildtype

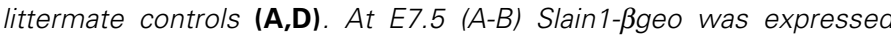
throughout the epiblast (B). At E9.5 (C-G) robust expression of Slain 1$\beta$ geo was observed along the length of the neural tube ( $n t)$ from forebrain (fb) to tailbud ( $t b$ ) with lower levels observed within the branchial arches (ba) and gut tube (gt) (D) but not in the heart (ht). Intense X-gal staining was also observed in the midbrain (mb), hindbrain (hb), otic vesicle (ot) and tailbud (E,F) and the dorsal root ganglia (drg) (G) 


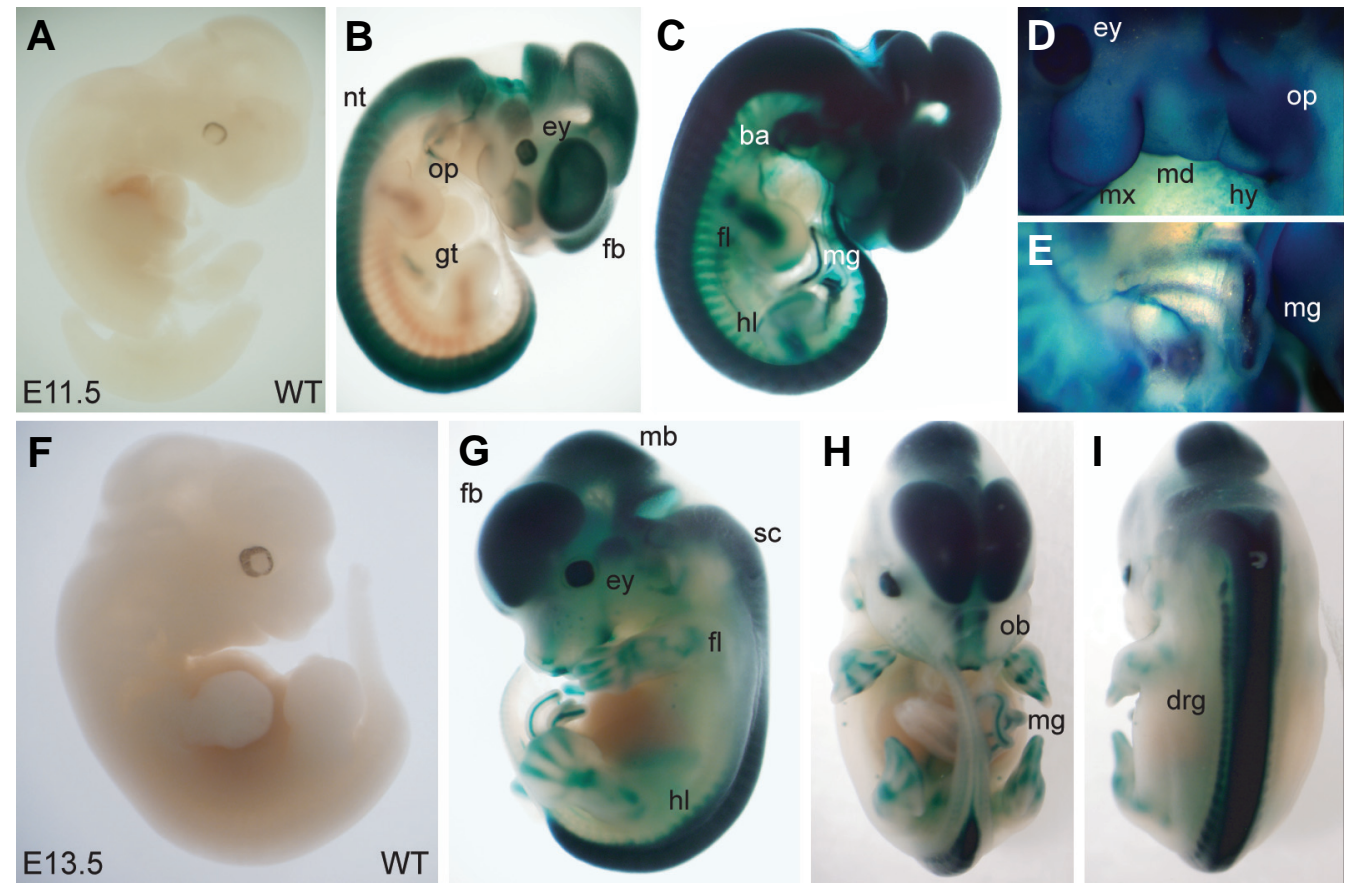

Fig. 3. Slain 1-Bgeo expression in the E11.5 and E13.5 embryo. Photomicrographs of whole mount $X$-gal stained Slain 1- $\beta$ geo homozygous mouse embryos (B-E, G-I) or wildtype littermate controls (A,F). At E11.5 (A-E) Slain 1$\beta$ geo expression was highest within the forebrain (fb), neural tube ( $n t$ ) and eye (ey) while fainter X-gal staining was evident within the oropharynx (op) and the gut tube (gt). (C) Prolonged staining of the same embryo as in (B) identified additional sites of expression within the branchial arches (ba) and midgut loop (mg) as well as the forelimb (fl) and hindlimb (hl). (D) Higher magnification indicates Slain 1- $\beta$ geo expression within the maxillary, mandibular and hyoid arches ( $m x$, md and hy respectively) overlying the oropharyngeal region. (E) Higher magnification of Slain 1- $\beta g$ eo expression in the midgut loop. At E13.5 (F-I), Slain1Bgeo expression was retained within the developing nervous system but was most prominent in the forebrain (fb), midbrain (mb) and spinal cord (sc) (G), the olfactory bulb (ob) (H) and the dorsal root ganglia (drg) (I). Slain 1-ßgeo expression was also retained within the developing forelimb and hindlimb (G) and in the midgut loop (H).

Bgeo expression in branchial arches, midgut loop and in the condensing mesenchyme of the developing limb buds (Fig. 3 CE). Embryos at E13.5 demonstrated extensive staining for Slain1$\beta$ geo throughout the developing nervous system, including the dorsal root ganglia and the olfactory bulb and within the endoderm derived midgut loop, in the physiological umbilical hernia (Fig. 3 G-I).

Sagittal sections of embryos at E11.5 and E13.5 confirmed the extensive expression of Slain $1-\beta g e o$ in neural tissue and revealed additional sites of expression in the main bronchi of the lung and within the developing metanephric tubules at E13.5 (Fig. $4 \mathrm{~A}, \mathrm{~B}$ and insert). Staining was observed within regions of the gastro-intestinal tract, particularly within the lumen of the midgut loop and the stomach at E11.5 (Fig. 4 A,B).

\section{Expression of Slain1- $\beta$ geo in the developing limb buds}

At E11.5, Slain1- $\beta$ geo expression was observed in the apical ectodermal ridge (AER) of the developing limb buds, the specialised region of surface ectoderm responsible for inducing growth and differentiation of the underlying limb mesenchyme (Fig. 5 A,B). At mid-gestation, development of the forelimb is more advanced than the hindlimb. The distal region of limb buds, which give rise to the hand plate, was marked by intense Slain $1-\beta g e o$ expression, while lower levels of expression were observed in the proximal region, which gives rise to the pectoral girdle and arm (Fig. 5C).

By E13.5 the hindlimb has developed the angular contours at its peripheral margin, which correspond to the future digits. The three central digital rays were marked by robust Slain $1-\beta g e o$ expression, whereas the two external digital rays had less intense $X$-gal staining (Fig. 5D). Additional Slain1- $\beta$ geo expression was apparent in the proximal region corresponding to the future tarsus (Fig. 5D). Similarly in the forelimb, robust Slain1- $\beta$ geo expression outlined the three central digits while lower levels marked the emerging digital rays corresponding to the two external digits. Furthermore, Slain $1-\beta g e o$ expression marked the future carpal and metacarpal bones (Fig. 5E). Slain1- $\beta$ geo expression was also observed within the maxillary surface elevations corresponding to the horizontal rows of vibrissae (Fig. 5E).

\section{Expression of Slain 1- $\beta g$ eo in the developing nervous system}

Robust Slain $1-\beta$ geo expression was detected in the neuroepithelium of mouse embryos examined at E9.5, E11.5 and E13.5. Transverse sections from the cranial region of an E9.5 embryo demonstrated extensive Slain $1-\beta g e o$ expression within the neuroepithelium surrounding the telencephalic, mesencephalic and optic vesicles. Slain $1-\beta g$ geo expression was also observed within condensations of cells corresponding to neural crest cells located on either side of the mesencephalic vesicle (Fig. 6A). Transverse sections from the mid-trunk region of an E9.5 embryo demonstrated extensive Slain1- $\beta$ geo expression within the neuroepithelium of the neural tube as well as neural crest derived cells located on either side of the neural tube, which give rise to the sensory cells of the dorsal root ganglia. Slain1-ßgeo expression was also observed in the hindgut diverticulum and the overlying surface ectoderm of the cranial region (Fig. 6 A,B).

Sagittal sections from the head region of an E11.5 embryo demonstrated pronounced Slain $1-\beta g e o$ expression within the neuroepithelium surrounding both the fourth and telencephalic ventricles. Higher magnification examination of the fourth and telencephalic ventricles indicated that Slain $1-\beta g e o$ expression was located within the ventricular zone however the level of expression varied between different stratified layers of the neuroepithelium. Slain $1-\beta g e o$ expression was absent from the roof of the hindbrain (Fig, 6 C-E).

Examination of histological sections of E13.5 X-gal-stained embryos indicated that Slain1- $\beta$ geo localised to the superficial 
A

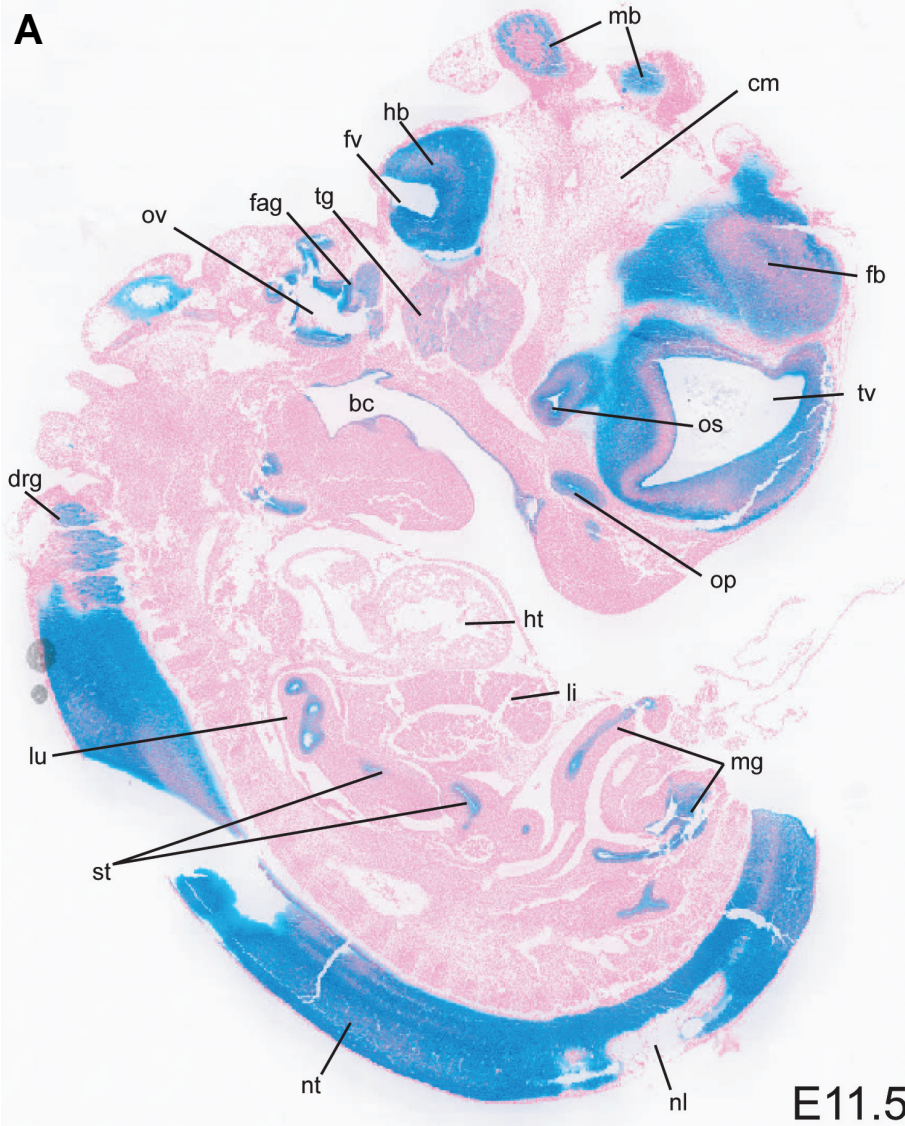

B

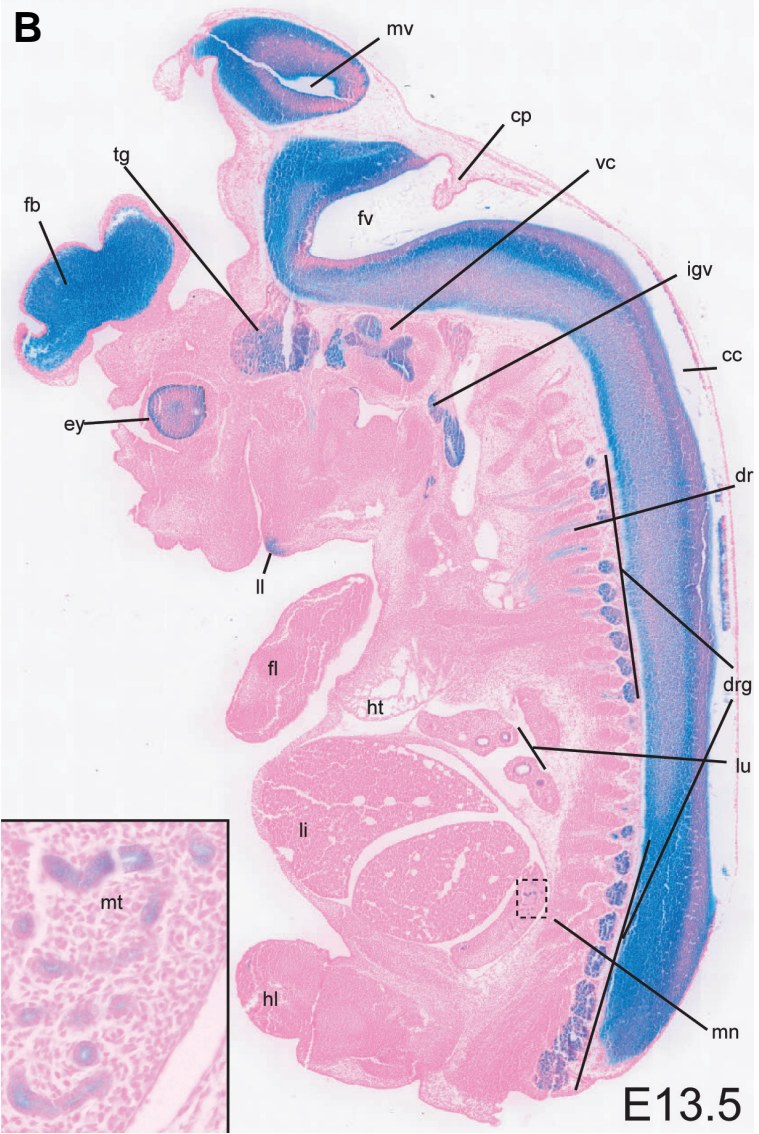

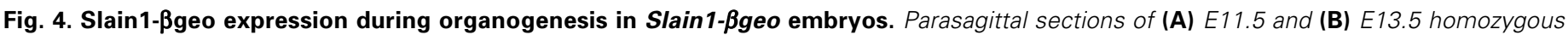
Slain 1-Bgeo embryos demonstrated $\beta$-galactosidase activity in the developing nervous system, as well as the lung, gut and metanephros (see insert) Abbreviations: bc, buccal cavity; cc, central canal; cm, cephalic mesenchyme; cp, choroid plexus; dr, dorsal rootlets of drg; drg, dorsal root ganglia; ey, eye; fag, facio-acoustic ganglion complex; fb, forebrain; fl, forelimb; fv, fourth ventricle; ht, heart; hb, hindbrain; hl, hindlimb; igv, inferior ganglion of the vagus nerve; li, liver; Il, lower lip; lu, lung; mn, metanephros; mb, midbrain; mg, midgut loop; mt, metanephric tubules; mv, mesencephalic vesicle; $n l$, lumen of the neural tube; nt, neural tube; op, olfactory pit; os, optic stalk; ov, otic vesicle; st, stomach; tg, trigeminal ganglion; tv, telencephalic vesicle $v c$, vestibulocochlear ganglion.
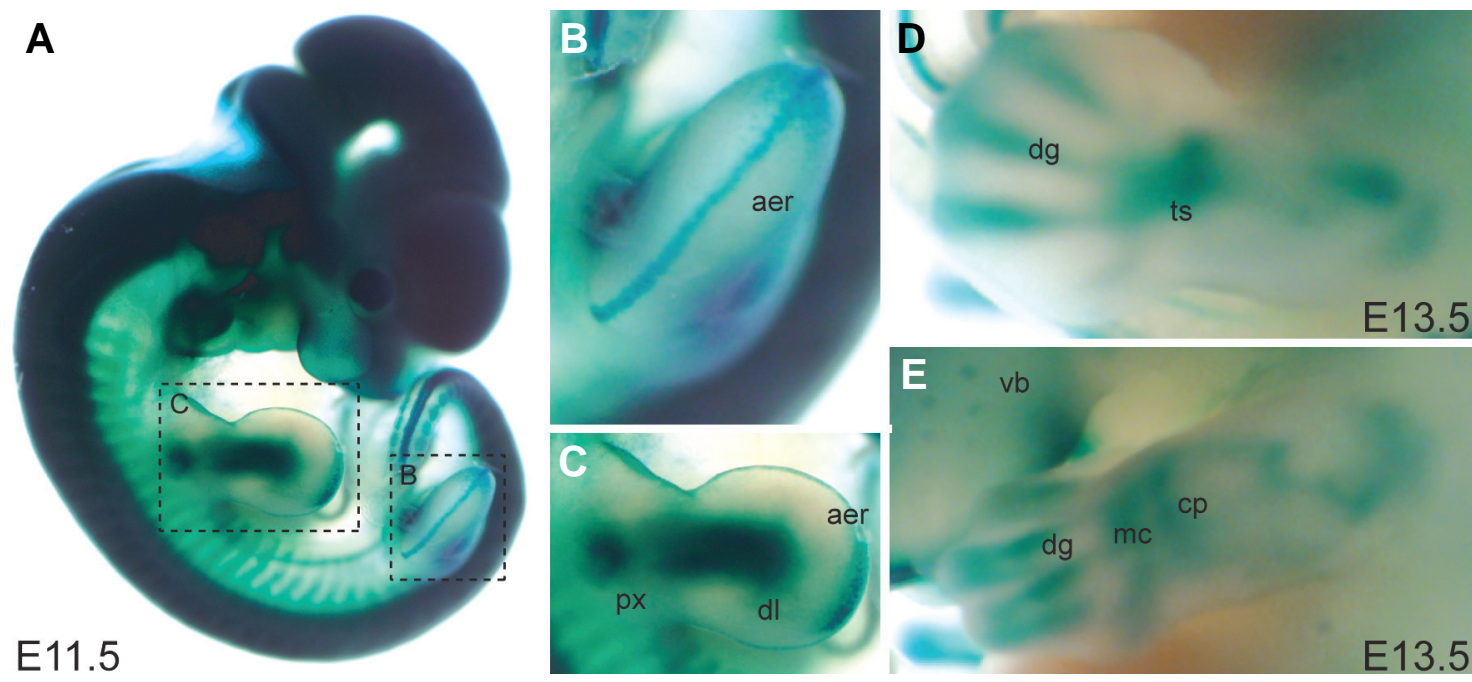

Fig. 5. Slain1-ßggeo expression in the developing limb bud at E11.5 and E13.5. Photomicrographs from wholemount X-gal stained Slain1-ßgeo homozygous mouse embryos at E11.5 (A-C) or E13.5

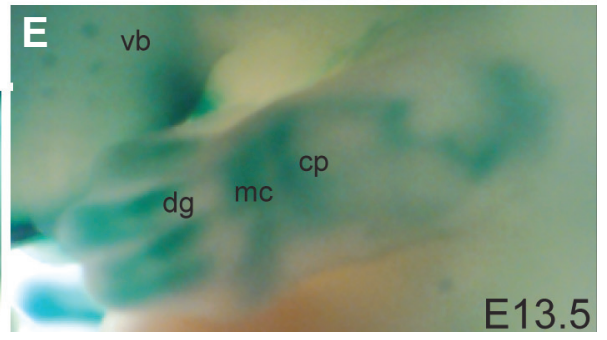
(D,E). AtE11.5, Slain 1- $\beta$ geo expression in the forelimb and hind limb was evident within the apical epidermal ridge (aer) and in the proximal (px) and distal (dl) regions of the condensing mesenchyme (B,C). At E13.5, X-gal staining marked the developing digits (dg) of the fore- and hindlimbs, and regions delineating the presumptive tarsal bones (ts) of the hindlimb (D), and the presumptive carpal (cp) and metacarpal ( $\mathrm{mc}$ ) bones of the forelimb (E). In addition Slain 1- $\beta g$ geo staining is present in the vibrissae (vb) on the maxillary region. 

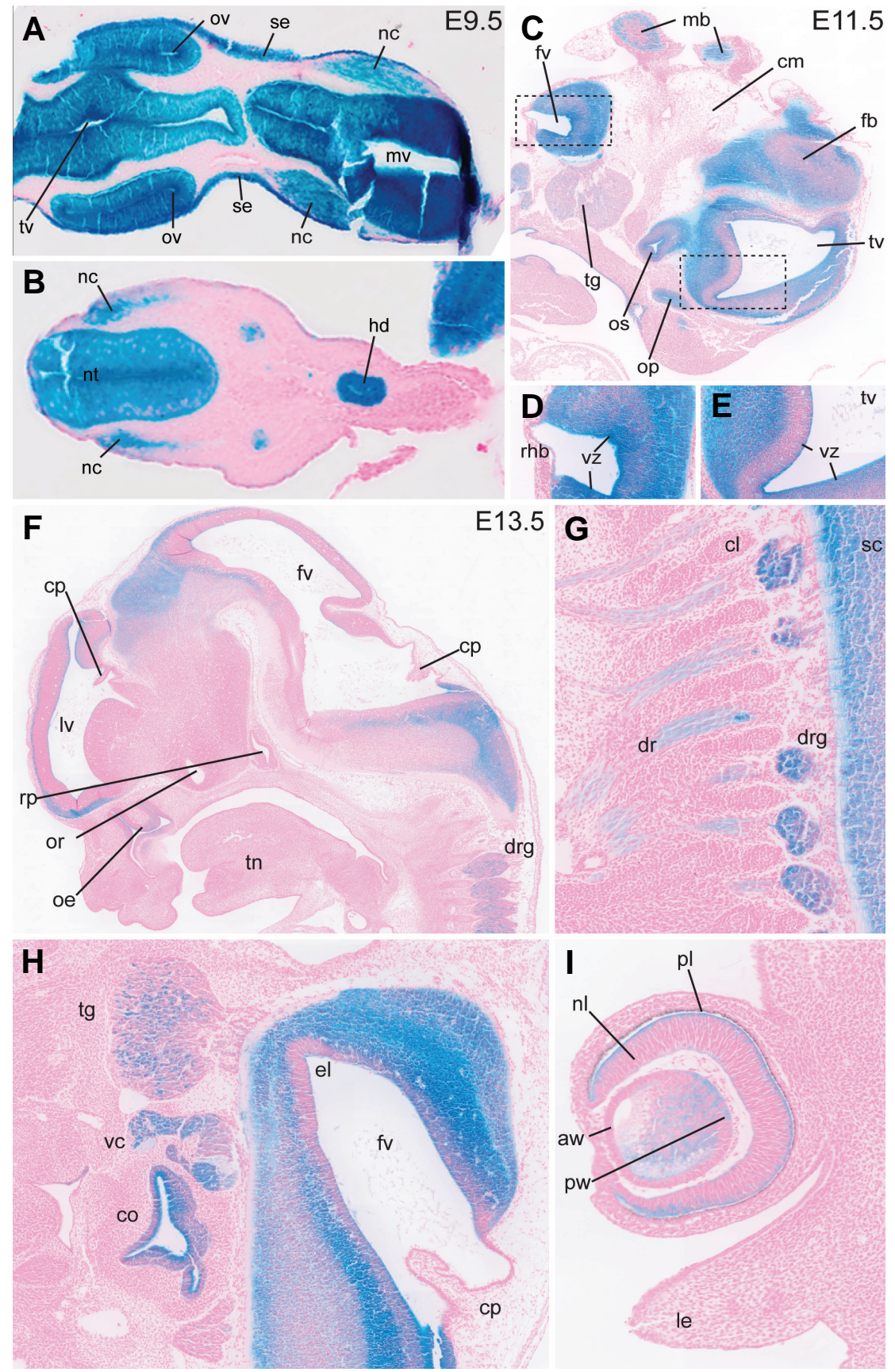

layers of the neuroepithelium and the ependymal layers surrounding the fourth and lateral ventricles (Fig. 6 F,H). Slain1- $\beta$ geo expression was apparent in the neurons of the dorsal root ganglia and in dorsal roots between the cartilage primordia (Fig. $6 G)$. X-gal staining was also observed in the cranial sensory ganglia, including the trigeminal ganglion, the facio-acoustic ganglion, the vestibulocochlear ganglion and the inferior ganglion of the vagus nerve (Figs. 4A, $B$ and $6 \mathrm{H}$ ). Examination of the sensory ganglia at $E 13.5$ revealed $X$-gal staining in both the neurons and in the neural projections emanating from the trigeminal ganglion and the vestibulocochlear ganglion towards the hindbrain (Fig. 6H).

In the developing sensory organs, Slain $1-\beta g e o$ expression was observed in the epithelium of the olfactory pit, optic stalk and otic vesicle at $E 11.5$ and the eye at E13.5 (Figs. 4 A,B and 6I). Histological analysis revealed $X$-gal staining throughout the epithelial layer of the cochlea, with the most pronounced expression associated with the apical surface (Fig. 6H). In the developing eye, Slain1$\beta$ geo expression localised to the elongated cells of the posterior wall of the lens vesicle and the posterior side of the neural layer.

This study has documented the developmental expression of a $\beta g e o$ gene trap vector inserted into the locus of the Slain 1 gene. $\beta$ geo expression was observed in distinct cell populations derived from all three germ layers, with highest levels associated with cells of the developing nervous system. This study of the Slain $1-\beta g e o$ mouse complements our previous work in which the expression of S/ain 1 was examined using ISH. Although it is important to bear in mind that the transgene reports only sites of gene expression, not endogenous protein localisation, use of the highly sensitive lac $Z$-based reporter system has allowed identification of additional sites in which Slain 1 may play a role. It is well recognized that random insertions of lac $Z$ transgenes may be associated with misexpression, in the form of silencing and variegated expression or ectopic sites of expression. It is believed that these are insertion site related phenomena that reflect the incomplete

Fig. 6. Expression of Slain1-ßggeo within the developing nervous system. (A,B) Transverse sections from an E9.5 embryo demonstrating Slain 1Bgeo expression within the neuroepithelium surrounding the telencephalic, mesencephalic and optic vesicles as well as in the neural tube (tv, mv, ov and nt), with additional Slain 1-Bgeo expression within the neural crest derived cells (nc) located on either side of the neural tube. Slain 1- $\beta$ geo expression was also observed in the overlying surface ectoderm (se) of the cranial region of the embryo (A) and in the hindgut diverticulum (hd) (B). (C) Sagittal sections of the head region from an E11.5 embryo (shown at low magnification in Figure 4A) demonstrating Slain 1-ßggeo expression within the neuroepithelium surrounding the fourth and telencephalic ventricles ( $f v, t v)$. cm, cephalic mesenchyme; fb, forebrain; $m b$, midbrain; op, olfactory pit; os, optic stalk; tg, trigeminal ganglion (D,E). Higher magnifications of the fourth and telencephalic ventricles demonstrating Slain 1- $\beta g$ geo expression

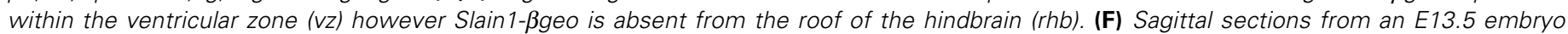
demonstrating Slain 1-Bgeo expression within the neuroepithelium surrounding the fourth and lateral ventricles (fv, IV), the dorsal root ganglia (drg) and the olfactory epithelium (oe). Expression was absent from the optic recess (or), Rathke's pouch (rp), intrinsic muscle of the tongue (tn) and choroid plexus (cp). (G) Sagittal section through the thoracic region demonstrating Slain 1-ßgeo expression within the drg, spinal cord (sc) and dorsal rootlets (dr). Expression was absent from the cartilage primordium (cl). (H) Magnified view of the area surrounding the fourth ventricle (fv) revealing expression within the ependymal layer (el), the trigeminal ganglion (tg), vestibulocochlear ganglion (vc) and cochlea (co). (I) Expression of Slain 1- $\beta g e o$ was also detected within the elongated cells of the posterior wall of the lens vesicle (pw) and neural layer (nl). Expression was not observed in the anterior wall of the lens vesicle (aw), the pigment layer (pl) or lower eyelid (le). 
regulatory sequences used to drive the transgene, as well as attributes of the lacZgene itself, such as transcriptional instability due to its high $\mathrm{G}+\mathrm{C}$ content (Montoliu et al., 2000). Interestingly, these insertion site effects are less evident when lac $Z$ is used as an embryonic reporter, especially in gene trap or knock in experiments. It is argued that more faithful expression patterns reflect the native configuration of the unperturbed regulatory sequences under these circumstances. Therefore, whilst we do not have multiple independent gene trap lines, it is still likely that the lacZ expression observed in the Slain 1- $\beta$ geo mouse faithfully reflects endogenous expression. Nevertheless, the generation of specific antibodies to Slain1 (including antibodies that differentiate between isoforms 1 and 2) will be required to fully elucidate the exact expression dynamics and localisation of the Slain1 protein in these tissues.

\section{Materials and Methods}

\section{Generation of Slain1- $\beta$ geo mice}

Mice were generated from the XG385 and XG752 mESC lines (Hirst etal., 2006) by Monash University Mouseworks transgenic facility (Monash University Animal Ethics Approval number - PHYS/MIS/2005/14). All mouse tissues were obtained post-mortem after euthanasia performed in accordance with Monash University guidelines. A candidate primer approach was performed to characterise the exact site of transgene integration, which indicated that both XG385 and XG752 have the same insertion point, suggesting they are sister clones. Genotyping of tail DNA was performed using primer pairs that flank the $\beta$ geo insertion point:

Geno F; 5'-TAGCCATTAGGACCTAGGACATGC-3' and

Geno R; 5'-TCTGGTCTGTGGCTTATCTGATCC-3' and

pß R; 5'-CTTCACTGAGTCTCTGGCATCTCC-3'.

\section{Analysis of splice variants}

Total RNA was extracted from adult mouse organs using the RNeasy Midi Kit (QIAGEN) and cDNA generated with SuperScript III (Invitrogen) according to the manufacturers' instructions. Semi-quantitative RT-PCR was performed as previously described (Elefanty et al., 1997) with the following primers

p2 F; 5'- CTCTTCAAAGACATTCACCTCACC-3',

p4 R; 5'- ATGACTGCGGACTAAGTGGAGAGC-3',

p $\beta \mathrm{R} ;$ 5'- CTTCACTGAGTCTCTGGCATCTCC-3',

HPRT F; 5'- GCTGGTGAAAAGGACCTCT-3' and

HPRT R; 5'- CACAGGACTAGAACACCTGC-3'.

\section{Visualisation of $\beta g e o$ expression using $X$-gal staining}

Whole embryos were fixed in $4 \%$ paraformaldehyde (PFA) in phosphate buffered saline (PBS) and then washed three times in washing buffer ( $0.1 \mathrm{M}$ phosphate buffer, $2 \mathrm{mM} \mathrm{MgCl}_{2}, 5 \mathrm{mM} \mathrm{EGTA}, 0.02 \%$ Nonidet$\mathrm{P} 40,0.01 \%$ sodium deoxycholate) at room temperature. Embryos were transferred to X-gal staining solution $\left(0.6 \mathrm{mg} / \mathrm{ml} \mathrm{X-gal,} 5 \mathrm{mM} \mathrm{K}_{4} \mathrm{Fe}(\mathrm{CN})_{6}\right.$, $5 \mathrm{mM} \mathrm{K}_{3} \mathrm{Fe}(\mathrm{CN})_{6}$ in wash buffer) and incubated at $37^{\circ} \mathrm{C}$ for $4 \mathrm{hr}$ or until colour developed. Stained embryos were washed in PBS with $3 \%$ DMSO before being post-fixed in $4 \%$ PFA/PBS at $4{ }^{\circ} \mathrm{C}$ overnight in order to preserve staining patterns. Embryos were photographed as whole mounts before embedding in paraffin for sectioning and histology. $10 \mu \mathrm{m}$ sections of embryos were counterstained with Nuclear Fast Red (Sigma).

\section{Acknowledgements}

This work was supported by the Australian Stem Cell Centre, the Juvenile Diabetes Research Foundation and the National Health and Medical Research Council of Australia. AGE is a Senior Research Fellow of the NHMRC.

\section{References}

ELEFANTY, A.G., ROBB, L., BIRNER, R. AND BEGLEY, C.G., (1997). Hematopoietic-specific genes are not induced during in vitro differentiation of scl-null embryonic stem cells. Blood90: 1435-1447.

HIRST, C.E., NG, E.S., AZZOLA, L., VOSS, A.K., THOMAS, T., STANLEY, E.G. AND ELEFANTY, A.G., (2006). Transcriptional profiling of mouse and human ES cells identifies SLAIN1, a novel stem cell gene. Dev. Biol. 293: 90-103.

MONTOLIU, L., CHAVEZ, S. AND VIDAL, M., (2000). Variegation associated with lacZ in transgenic animals: a warning note. Transgenic Res 9: 237-239. 


\section{Further Related Reading, published previously in the Int. J. Dev. Biol.}

See Special Issue Pattern Formation edited by Michael K. Richardson and Cheng-Ming Chuong at:

http://www.ijdb.ehu.es/web/contents.php?vol=53\&issue=5-6

Ectopic Meis1 expression in the mouse limb bud alters P-D patterning in a Pbx1-independent manner Nadia Mercader, Licia Selleri, Luis Miguel Criado, Pilar Pallares, Carlos Parras, Michael L. Cleary and Miguel Torres Int. J. Dev. Biol. (2009) 53: 1483-1494 (doi: 10.1387/ijdb.072430nm)

The surface ectoderm of the chick embryo exhibits dynamic variation in its response to neurogenic signals Vineeta-Bhasker Tripathi, Yasuo Ishii, Muhammad M. Abu-Elmagd and Paul J. Scotting Int. J. Dev. Biol. (2009) 53: 1023-1033

Limb pattern, physical mechanisms and morphological evolution - an interview with Stuart A. Newman

Cheng-Ming Chuong

Int. J. Dev. Biol. (2009) 53: 663-671

Generation of a reporter-null allele of Ppap2b/Lpp3and its expression during embryogenesis Diana Escalante-Alcalde, Sara L. Morales and Colin L. Stewart

Int. J. Dev. Biol. (2009) 53: 139-147

The preplacodal region: an ectodermal domain with multipotential progenitors that contribute to sense organs and cranial sensory ganglia

Andrea Streit

Int. J. Dev. Biol. (2007) 51: 447-461

Analysis of a new allele of limb deformity (Id) reveals tissue- and age-specific transcriptional effects of the Ld Global Control Region

Emilia Pavel, Wenning Zhao, Kimerly A. Powell, Michael Weinstein and Lawrence S. Kirschner Int. J. Dev. Biol. (2007) 51: 273-281

Postimplantation mouse embryos cultured in vitro. Assessment with whole-mount immunostaining and in situ hybridization

G Van Maele-Fabry, F Clotman, F Gofflot, J Bosschaert and J J Picard

Int. J. Dev. Biol. (1997) 41: 365-374

Ectopic Meis1 expression in the mouse limb bud alters $\mathrm{P}-\mathrm{D}$ patterning in a Pbx1-independent manner

Nadia Mercader, Licia Selleri, Luis Miguel Criado, Pilar Pallares, Carlos Parras, Michael L. Cleary and Miguel Torres

Int. J. Dev. Biol. in press (doi: 10.1387/ijdb.072430nm)

Expression of csal1 in pre limb-bud chick embryos

Dylan Sweetman, Terence G. Smith, Elizabeth R. Farrell and Andrea Münsterberg

Int. J. Dev. Biol. (2005) 49: 427-430

The mouse polydactylous mutation, luxate (Ix), causes anterior shift of the anteroposterior border in the developing hindlimb bud

Yukari Yada, Shigeru Makino, Sadao Chigusa-Ishiwa and Toshihiko Shiroishi

Int. J. Dev. Biol. (2002) 46: 975-982

Distribution and possible function of an adrenomedullin-like peptide in the developing chick limb bud

M Reza Seghatoleslami, Alfredo Martínez, Frank Cuttitta and Robert A Kosher

Int. J. Dev. Biol. (2002) 46: 957-961

Role of chondrogenic tissue in programmed cell death and BMP expression in chick limb buds

M Omi, M Sato-Maeda and $\mathrm{H}$ Ide

Int. J. Dev. Biol. (2000) 44: 381-388

Neural crest cell migration and pigment pattern formation in urodele amphibians H H Epperlein, J Löfberg and L Olsson

Int. J. Dev. Biol. (1996) 40: 229-238

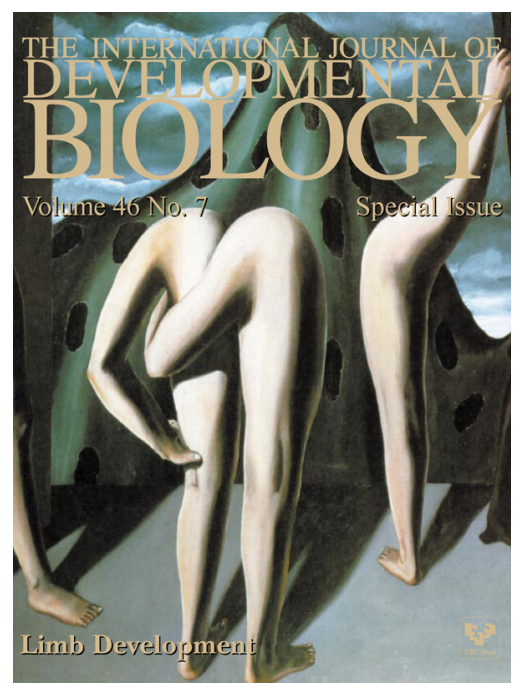

5 yr ISI Impact Factor $(2009)=3.253$

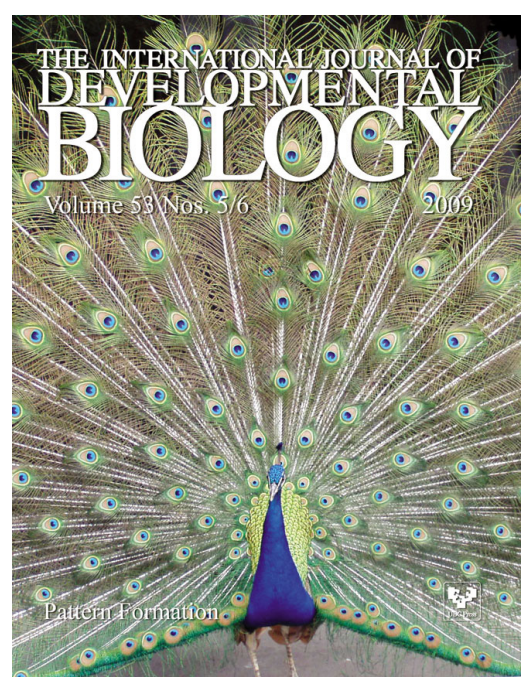

